\title{
Controle ácido-básico na hipotermia
}

\author{
Walter José GOMES*, Ênio BUFFOLO*
}

RBCCV 44205-214

GOMES, W. J. \& BUFFOLO, E. - Controle ácido-básico na hipotermia. Rev. Bras. Cir. Cardiovasc., 8 (3): 189-194, 1993.

\begin{abstract}
RESUMO: O emprego da hipotermia profunda tem se constituído, atualmente, numa importante estratégia para melhoria da qualidade técnica e resultados em cirurgia cardiovascular. A hipotermia reduz os danos teciduais induzidos pela isquemia por diminuir o metabolismo e preservar os fosfatos energéticos. A regulação do $\mathrm{pH}$ tecidual durante a hipotermia é fundamental para a manutenção da homeostasia celular, já que a hipotermia induz alteraçōes desse pH pela mudança provocada na constante de dissociação da água. A questão do melhor manuseio dos gases sangüineos durante a hipotermia induzida tem sido objeto de controvérsia. Duas abordagens têm sido preconizadas para o manejo das alterações iônicas durante a hipotermia. A regulção $\mathrm{pH}$-stat envolve a manutenção do $\mathrm{pH}$ constante de $7,40 \mathrm{em}$ todas as temperaturas com ajustes da $\mathrm{PaCO}_{2}$ e a regulação $\alpha$-stat permite a variação do $\mathrm{pH}$ sangūíneo, que aumenta conforme a diminuição da temperatura e o conteúdo total corpóreo de $\mathrm{CO}_{2}$ é mantido constante. Nesta presente revisão a relação entre $\mathrm{pH}$ sangūineo e intracelular e as alteraçōes iônicas induzidas pela hipotermia são discutidas.
\end{abstract}

DESCRITORES: hipotermia induzida, em cirurgia cardiaca.

A hipotermia profunda tem sido utilizada como um importante adjunto em cirurgia cardiaca, especialmente associado com a circulação extracorpórea. Essa utilidade tem aumentado atualmente, devido ao aumento de complexidade das operações e necessidade freqüente de abordagem do arco aórtico para correçōes a esse nível $8,14,35$.

O emprego da hipotermia profunda é baseado na redução do metabolismo tecidual com a queda da temperatura, como descrito pela teoria de Arrhenius, mostrando a relação de temperatura com a velocidade das reações químicas, reduzindo, assim, o risco de dano isquêmico durante as operações 10 .

Apesar dessa intensa utilização, o manejo das alterações ácido-básicas induzidas pela hipotermia permanece controvertida. STADIE et alii ${ }^{34}$ e AUSTIN \& CULLEN 1, em 1925, mostraram que, num sistema fechado, quando o conteúdo de $\mathrm{CO}_{2}$ é mantido constante, o $\mathrm{pH}$ do plasma aumenta conforme a temperatura é abaixada. O uso de valores, corrigidos ou não, dos gases sangüíneos denominados, respectivamente, regulação $\mathrm{pH}$-stat e $\alpha$-stat, resultam das alterações iônicas que aparecem com as variações corpóreas de temperatu-ra 2, 26, 36, 39 . 42,43 .

Essas duas abordagens têm sido utilizadas. A primeira abordagem consiste em ajustar a pressão parcial arterial de $\mathrm{CO}_{2}\left(\mathrm{PaCO}_{2}\right)$ para manter o sangue com pH próximo de 7,40 em todas as temperaturas. Isto é denominado sistema $\mathrm{pH}$-stat, porque procura manter um $\mathrm{Ph}$ arterial constante com qualquer variação de temperatura do paciente. Para isto, há necessidade de se acrescentar dióxido de carbono ao sangue a fase de esfriamento do paciente em circulação extracorpórea 4, 21. A outra abordagem, conhecida como sistema $\alpha$-stat (assim chamado por causa da constante de dissociação do grupo $\alpha$-imidazólico do aminoácido histidina), como preconizado por RAHN et alii ${ }^{24,25}$ e REEVES ${ }^{28}$, mantém constante o conteúdo total de $\mathrm{CO}_{2}$ do sangue, permitindo a variação de $\mathrm{pH}$ com o esfriamento. 
Este conceito de regulação tornou-se usual na prática clínica em todo o mundo nos últimos 10 anos, principalmente pelas evidências que o consumo total de oxigênio corpóreo é maior no $\alpha$-stat que no $\mathrm{pH}$ stat, o que pressupõe melhor preservação metabólica 12,17 .

Tradicionalmente, os valores sangüíneos de $\mathrm{pH}=7,40$ e $\mathrm{PaCO}_{2}=40 \mathrm{mmHg}$, em normotermia são ditos "normais", mas esses conceitos não podem ser extendidos quando o paciente se encontra em hipotermia.

Os conceitos de regulação ácido-básica desenvolvidos após os trabalhos de SEVERINGHAUS 32,33 e os nomogramas para correção dos gases sangüíneos arteriais com a temperatura conduziram ao uso de valores normais corrigidos de $\mathrm{pH}$ e $\mathrm{PCO}_{2}$ nas primeiras duas décadas do uso da hipotermia (sistema pH-stat). Em hipotermia, como há menor produção de $\mathrm{CO}_{2}$ pela diminuição do metabolismo basal e também aumento da solubilidade do $\mathrm{CO}_{2} 6,31$, era administrado $\mathrm{CO}_{2}$ com o propósito de aumentar - $\mathrm{CO}_{2}$ corpóreo total para manter esses valores corrigidos "normais". Há de se salientar que os normogramas têm valor limitado para correção desses valores em circulação extracorpórea porque a hemodiluição altera significativamente o hematócrito, concentração de proteínas e eletrólitos.

O sistema $\alpha$-stat teve incremento na utilização clínica em circulação extracorpórea hipotérmica a partir da década de 80 , após os estudos de HOWELL et alii ${ }^{11}$ e RAHN et alii ${ }^{24,25}$ e REEVES ${ }^{27-31}$, em animais ectodérmicos e poicilotérmicos, desenvolvendo um conceito de regulação ácido-básica aplicável a peixes, répteis e anfíbios que apresentam grandes variações de temperatura corpórea. Este conceito é baseado na manutenção da neutralidade eletroquímica intracelular em todas as temperaturas.

A estrutura molecular e a função das proteínas, principalmente os sistemas enzimáticos, dependem de um ambiente eletroquímico neutro, pois as cargas eletroquímicas e as ligações mantêm a estrutura molecular das proteinas. Os sistemas de regulação ácido-básicos devem proporcionar um meio intracelular neutro, no qual o número de íons $\mathrm{H}^{+}$e $\mathrm{OH}^{-}$sejam iguais ${ }^{7}$, praticamente $1: 1$.

$\mathrm{O} \mathrm{pH}$ é calculado pelo logarítmo negativo da concentração hidrogênio-iônica $\left(\left[\mathrm{H}^{+}\right]\right)$em soluções aquosas, como a célula ou o sangue, onde a água é a fonte primária de $\mathrm{H}^{+}$. A constante de dissociação da água $\left(\mathrm{pK}_{\mathrm{H} 2 \mathrm{O}}\right)$ controla $\mathrm{opH}$ e aumenta com 0 decréscimo da temperatura. Portanto, o número de íons $\mathrm{H}^{+}$diminui quando, em hipotermia e, conseqüentemente, $\circ \mathrm{pH}$, que é o logarítmo negativo da $\left[\mathrm{H}^{+}\right]$aumenta. Em soluções neutras, mesmo em hipotermia, o número de íons $\mathrm{OH}$-, que também é regulado pelo $\mathrm{pK}_{\mathrm{H} 2 \mathrm{O}}$, acompanha o de $\mathrm{H}^{+}$, mantendo uma relação constante 1:1. Então, o pH de neutralidade eletroquímica muda com a temperatura, mas a relação $\mathrm{H}^{+} / \mathrm{OH}^{-}$mantém-se constante.

No Gráfico 1 observamos que, com a mudança de temperatura, a faixa de $\mathrm{pH}$ do sangue mantém-se paralela à dos tecidos, com uma diferença média em pH entre 0,6 e $0,8(\mathrm{~K})$ unidades de $\mathrm{pH}$. O sangue mantém uma alcalinidade relativa constante em relação aos tecidos $\left(\mathrm{pH}_{\mathrm{i}}\right)$ em todas as temperaturas biológicas e o $\mathrm{pH}$ varia de conformidade com o $\mathrm{pK}_{\mathrm{H} 2 \mathrm{O}}$.

A concentração hidrogênio-iônica expressa em termos de $\mathrm{pH}$ e $\mathrm{pOH}$ tem a seguinte relação quanto ao ponto neutro da água $(\mathrm{pN})$ :

$$
\begin{aligned}
& \mathrm{pN}=\frac{\mathrm{pH}+\mathrm{pOH}}{2}=0,5 \mathrm{pK}_{\mathrm{H} 2 \mathrm{O}}, \text { onde } \\
& \mathrm{pN}=\mathrm{pH} \text { de neutralidade } \\
& \mathrm{pK}_{\mathrm{H} 2 \mathrm{O}}=\begin{array}{l}
\text { constante de dissociação da água a } \\
\text { uma dada temperatura }
\end{array} \\
& \mathrm{pOH}=\left[\mathrm{OH}^{-}\right]
\end{aligned}
$$

O sistema enzimático depende de um $\mathrm{pH}$ intracelular $(\mathrm{pHi})$ ótimo para seu funcionamento adequado. A neutralidade eletroquímica $\left(\left[\mathrm{H}^{+}\right] /\left[\mathrm{OH}^{-}\right]=1\right)$ proporciona que proteínas, enzimas e eletrólitos permaneçam em estado ionizado, mantendo o meta-

\section{GRÁFICO 1}

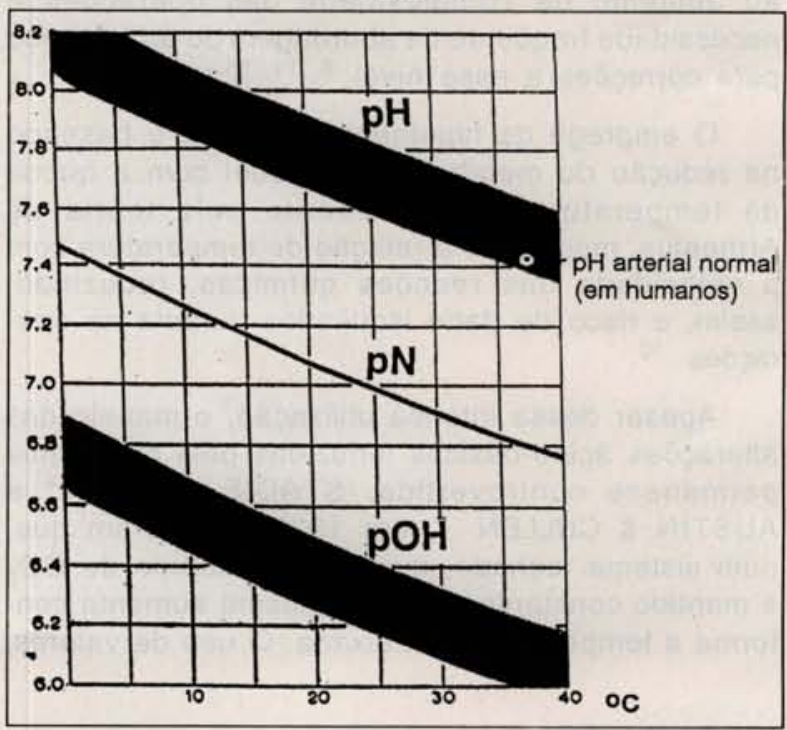

Valores de $\mathrm{pH}$ e $\mathrm{pOH}$ do sangue arterial de vertebrados obtidos a várias temperaturas. É mantida constante a diferença entre $\mathrm{opH}$ sanguíneo e o pN ( $\mathrm{pH}$ de neutralidade química da água) para cada nivel de temperatura. A diferença é de 0,6 a 0,8 unidades de $\mathrm{pH}$ e a relação $\left[\mathrm{H}^{+}\right] /\left[\mathrm{OH}^{-}\right]$permanece constante. 
bolismo e a estabilidade celular, já que compostos ionizados têm menor tendência em se difundir através da membrana celular. A ionização completa é um mecanismo eficiente de retenção de metabólitos dentro das células ou das organelas. Quando há aumento da concentração de íons $\mathrm{H}^{+}$, esses metabólitos perdem seus estados ionizados e passam a nãoionizados, que podem atravessar a membrana celular lipídica e alterar as concentrações e gradientes intracelulares, levando a menor produção de fosfatos energéticos e a alteração do conteúdo de água intracelular. A preservação do $\mathrm{pH}$ intracelular neutro e a manutenção da neutralidade eletroquímica mantêm a funçāo celular e a sua viabilidade, preservando os fosfatos energéticos, concentração de metabólitos, estrutura e função de proteínas e enzimas, concentração iônica, volume celular e o equilíbrio de Donnan ${ }^{30}$.

Para que a estabilidade intracelular seja conseguida, é necessário a regulação do meio externo que banha as células e os tecidos. $\mathrm{O} \mathrm{pH}$ intracelular normal oscila entre 6,8 e 6,9 a $37^{\circ} \mathrm{C}$ e implica numa concentração equalitária de íons $\mathrm{H}^{+} \mathrm{e}$ $\mathrm{OH}^{-}$, na proporção aproximada de 1:1. O meio extracelular é relativamente alcalino em relação ao intracelular, com $\mathrm{pH}$ ao redor de 7,4. Para manter este $\mathrm{pH}$, a relação de concentração entre $\mathrm{H}^{+}$e $\mathrm{OH}^{-}$é de aproximadamente 1:16. Este gradiente hidrogênioiônico entre os meios intra e extracelular é essencial porque permite que os produtos ácidos e $\mathrm{CO}_{2}$ resultantes do metabolismo celular sejam descarregados no meio extracelular.

A dissociação da água é profundamente alterada pela temperatura. Em hipotermia a $20^{\circ} \mathrm{C}$, com o aumento da constante de dissociação da água, a manutenção da neutralidade eletroquímica intracelular $\left(\left[\mathrm{H}^{+}\right]=\left[\mathrm{OH}^{-}\right]\right)$é conseguido com um $\mathrm{pH}$ ao redor de 7,1 . Por conseguinte, o meio extracelular, para manter-se relativamente alcalino, precisa manter um $\mathrm{pH}$ ao redor de $7,7\left(\left[\mathrm{H}^{+}\right]:\left[\mathrm{OH}^{-}\right]=1: 16\right)$. $\mathrm{O}$ comportamento do $\mathrm{pH}$ sangüíneo é regulado de maneira que, para qualquer temperatura, seja mais alcalino que $\circ \mathrm{pH}$ intracelular e $\circ \Delta \mathrm{pH}$ entre célula e sangue permaneça constante.

A diferença entre $\mathrm{opH}$ do meio extracelular e o da neutralidade $(\mathrm{pN})$ varia entre 0,6 e 0,8 unidades de $\mathrm{pH}$, que é designado como k. Então, a qualquer temperatura a concentração intracelular de $\mathrm{H}^{+}$é representada pelo $\mathrm{pN}$ e o do meio extracelular por $\mathrm{pN}+\mathrm{k}$.

Os sistemas tampões são responsáveis pela manutenção do $\mathrm{pH}$ e o principal sistema tampão envolvido é representado pelas proteínas, mais precisamente pelo grupo imidazólico presente no aminoácido histidina, que faz parte de muitas proteínas.
Com a queda de temperatura, o tampão proteico imidazólico muda seu pK (constante de dissociação iônica) em paralelo com o pN da água. Por causa desse paralelismo, o $\mathrm{pH}$ intracelular e também o $\mathrm{pH}$ sangüíneo aumentam com a diminuição da temperatura, enquanto $\circ \mathrm{CO}_{2}$ corpóreo total permanece igual. Contudo, com o aumento da solubilidade dos gases proporcionado pela hipotermia, a $\mathrm{PaCO}_{2}$ sangüínea diminui mas a fração não ionizada do anel $\alpha$-imidazólico presente nas proteínas permanece constante nessa situação. Isso significa que a carga elétrica total desse tampão permanece inalterado com a mudança de temperatura e de $\mathrm{pH}$.

Em todos os animais, os compartimentos intracelular e extracelular possuem mecanismos tampões formados pela combinação de ácidos fracos, carbonatos, fosfatos e proteínas. No fluido extracelular, entretanto, o tampão fosfato tem tão pouca ação tamponante que em termos práticos o meio extracelular é considerado um sistema binário de bicarbonato e proteína. Este complexo sistema age para proteger o $\mathrm{pH}$ intracelular em todas as temperaturas e embora todos estes componentes contribuam para o resultado, o mais importante na pre-

GRÁFICO 2

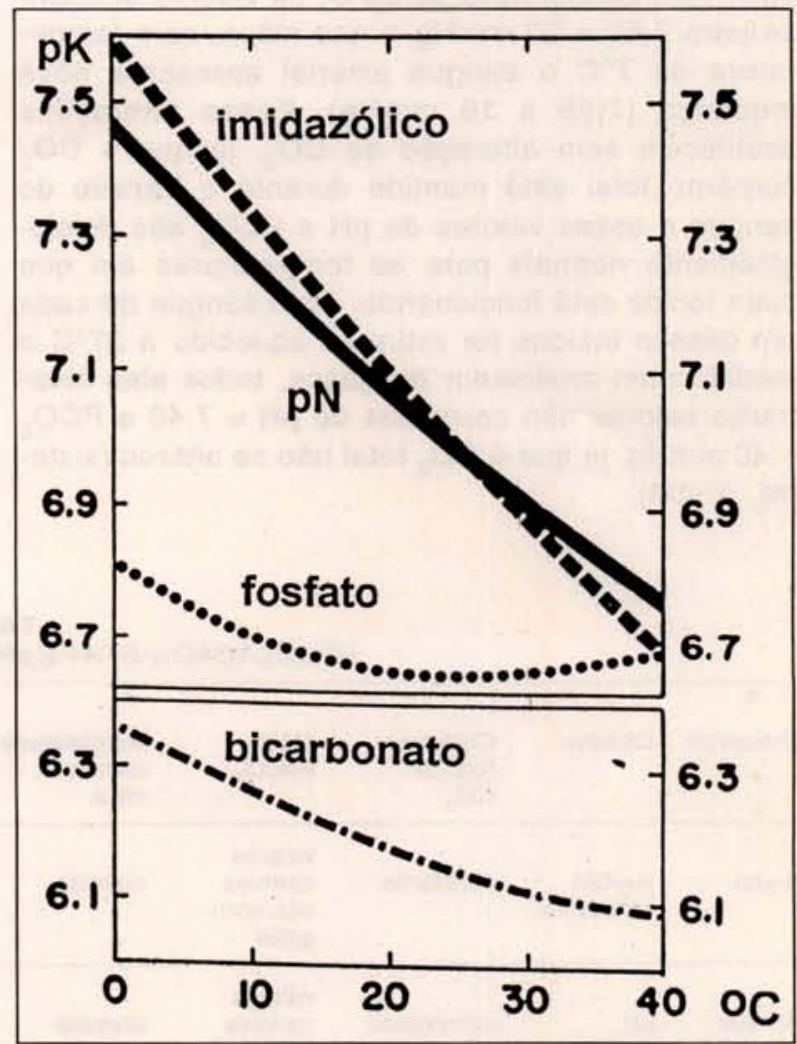

Alterações induzidas pela temperatura no pK dos tampões bicarbonato, fosfato e imidazólico, comparado com o pN. Fica claro que apenas o grupo imidazólico se aproxima das alterações observadas no pN. 
servação do balanço é o grupo imidazólico, devido ao comportamento do seu pK com as alterações de temperatura. O comportamento térmico do $\mathrm{pK}$ dessa proteína torna-a o mais importante mecanismo tampão contra as variações de temperatura nos vertebrados, como mostrado no Gráfico 2.

O equilibrio de Donnan pode ser alterado quando a $37^{\circ} \mathrm{C}$ ocorrem alteraçōes de $\mathrm{pH}$ intracelular, levando a uma redistribuição de eletrólitos entre a célula e o plasma, podendo alterar o volume das células por acúmulo de água. $\mathrm{Na}$ hipotermia, esse equilíbrio não é alterado, já que a concentração relativa de $\mathrm{Cl}^{-}$e $\mathrm{HCO}_{3}$ - entre célula e plasma permanece inalterado e não ocorre mudança de volume celular.

RAHN 24 assinala que o sangue arterial em humanos normotérmicos representa uma heterogeneidade de $\mathrm{pHs}$, dependendo da temperatura dos vários tecidos. Numa pessoa, exercitando-se num dia frio, o sangue arterial ejetado do coração tem valores "normais" de pH e $\mathrm{PCO}_{2}(7,40$ e $40 \mathrm{mmHg}$, respectivamente), mas sofrerá alteração térmica dependendo do tecido periférico, antes que ocorra a troca a nivel capilar. Quando este sangue chega ao músculo exercitando a $42^{\circ} \mathrm{C}$ estes valores mudam para preservar o alfa $(7,35$ e $47 \mathrm{mmHg})$. $\mathrm{Na}$ pele, com temperatura de $25^{\circ} \mathrm{C}$, os valores alteramse para 7,60 e $23 \mathrm{mmHg}$ e nas mãos, com temperatura de $7^{\circ} \mathrm{C} \circ$ sangue arterial apresenta nova mudança $(7,66$ e $16 \mathrm{mmHg})$. Essas alterações acontecem sem alteração do $\mathrm{CO}_{2}$, já que $\circ \mathrm{CO}_{2}$ corpóreo total está mantido durante o trânsito do sangue e esses valores de $\mathrm{pH}$ e $\mathrm{PCO}_{2}$ são fisiologicamente normais para as temperaturas em que cada tecido está funcionando. Se o sangue de cada um desses tecidos for retirado, aquecido a $37^{\circ} \mathrm{C}$ e medido num analisador de gases, todos eles mostrarão valores não corrigidos de $\mathrm{pH}=7,40$ e $\mathrm{PCO}_{2}$ $=40 \mathrm{mmHg}$, já que $\circ \mathrm{CO}_{2}$ total não se alterou (sistema $\alpha$-stat).
Dúvidas sobre qual o melhor mecanismo de regulação iônica na hipotermia permanecem ${ }^{3}, 5,13$, $19,20,23$, embora existam evidências, atualmente, da superioridade do manejo $\alpha$-stat $16,37,38,41$, desde que em muitas espécies biológicas estudadas (humanos, répteis, anfíbios, mamíferos hibernantes) - pHi se comporta muito próximo ao sistema $\alpha$ stat $25-27,36,43$.

As duas estratégias produzem diferentes ajustes na auto-regulação, isto é, no fluxo sangüineo cerebral (FSC) e no metabolismo durante a circulação extracorpórea hipotérmica não-pulsátil. As abordagens $\mathrm{pH}$-stat e $\alpha$-stat são comparadas na Tabela 1.

Autores têm demonstrado que na regulação $\mathrm{pH}$ stat, que mantém o pH intracelular acidótico, há alteração na atividade de enzimas e proteínas, com maior depressão metabólica ${ }^{22}$. O fluxo sangüíneo cerebral está aumentado 1,5 a 2 vezes por causa do aumento da $\mathrm{PaCO}_{2}{ }^{17}, 18$, com possível melhor perfusão cerebral, mas há o risco de hiperemia com aparecimento de edema cerebral, hipertensão intracraniana e de aumento de embolia por debris para o cérebro em relação à circulação sistêmica (desacoplamento fluxo/necessidade metabólica) 3 , 16. No sistema $\alpha$-stat há atividade enzimática normal e fluxo sangüineo cerebral reduzido, mas este fluxo pode ser 0 apropriado, já que há redução de consumo de oxigênio cerebral por menor demanda metabólica (acoplamento fluxo/necessidade metabólica). Também há o desvio para a esquerda da curva de dissociação da orci-hemoglobina, com conseqüente diminuição da oferta de oxigênio a nivel tecidual ${ }^{9}, 26,43$. Dados disponíveis no momento não permitem conclusões sobre nenhum desses conceitos hipotéticos.

Está claro que serão necessários estudos mais profundos sobre a melhor relação de controle ácidobásico entre os meios intra e extra-celular para procurar solver o problema de melhor manuseio hidrogênio-iônico na hipotermia.

TABELA 1

REGULAÇÃO $\alpha$-STAT E PH-STAT DURANTE HIPOTERMIA

\begin{tabular}{|c|c|c|c|c|c|c|c|c|c|}
\hline Estratégia & Objetivo & $\begin{array}{l}\text { Conteúdo } \\
\text { total de } \\
\mathrm{CO}_{2}\end{array}$ & $\begin{array}{l}\mathrm{pHe} \\
\mathrm{PaCO}_{2}\end{array}$ & $\begin{array}{l}\text { Neutralidade } \\
\text { eletroqui- } \\
\text { mica }\end{array}$ & $\begin{array}{l}\alpha \text {-imida- } \\
\text { zólico }\end{array}$ & $\begin{array}{l}\text { Estrutura } \\
\text { proteica e } \\
\text { funçāo } \\
\text { enzimática }\end{array}$ & $\begin{array}{l}\text { Fluxo } \\
\text { sangüíneo } \\
\text { cerebral } \\
\text { (FSC) }\end{array}$ & $\begin{array}{l}\text { Relaçāo } \\
\text { FSC/meta- } \\
\text { bolismo }\end{array}$ & $\begin{array}{l}\text { Efeito no } \\
\text { tecido } \\
\text { isquêmico }\end{array}$ \\
\hline$\alpha$-stat & $\begin{array}{l}\mathrm{H}+/ \mathrm{OH}- \\
\text { constante }\end{array}$ & constante & $\begin{array}{l}\text { valores } \\
\text { normais } \\
\text { nāo-corri- } \\
\text { gidos }\end{array}$ & mantida & $\begin{array}{l}\text { capacidade } \\
\text { tamponante } \\
\text { preservada }\end{array}$ & $\begin{array}{l}\text { normal e } \\
\text { atividade } \\
\text { máxima }\end{array}$ & $\begin{array}{l}\text { diminuido } \\
\text { (mas pro- } \\
\text { porcional?) }\end{array}$ & $\begin{array}{l}\text { fluxo e me- } \\
\text { tabolismo } \\
\text { acoplados? }\end{array}$ & $\begin{array}{l}\text { melhor } \\
\text { proteção } \\
\text { hipotér- } \\
\text { mica }\end{array}$ \\
\hline $\mathrm{pH}$-stat & $\begin{array}{l}\mathrm{pH} \\
\text { constante }\end{array}$ & aumentado & $\begin{array}{l}\text { valores } \\
\text { normais } \\
\text { corrigidos } \\
\text { para tem- } \\
\text { peratura }\end{array}$ & alterada & $\begin{array}{l}\text { diminuição } \\
\text { da } \\
\text { capacidade } \\
\text { tamponante }\end{array}$ & $\begin{array}{l}\text { alterada e } \\
\text { atividade } \\
\text { reduzida }\end{array}$ & inalterado & $\begin{array}{l}\text { fluxo exces- } \\
\text { sivo para o } \\
\text { metabolis- } \\
\text { mo? }\end{array}$ & $\begin{array}{l}\text { pior } \\
\text { proteção } \\
\text { hipotér- } \\
\text { mica? }\end{array}$ \\
\hline
\end{tabular}


GOMES, W. J. \& BUFFOLO, E. - The acid-base management in hypothermia. Rev. Bras. Cir. Cardiovasc., $8(3): 189-194,1993$.

ABSTRACT: Deep hypothermia is a usefull tool to improve technical results in cardiovascular surgery and is nowadays the major strategy used to reduce ischemic injury. Hypothermia reduces metabolism and preserves cellular stores of high-energy phosphates. The regulation of tissue $\mathrm{pH}$ during hypothermia is important for cellular homeostasis. Furthermore, hypothermia has important effects on $\mathrm{pH}$ by altering the dissociation constant of water and various metabolics intermediates and the question of optimal blood gas management during deliberate hypothermia has been subject of much controversy. Two approaches have been advocated for $\mathrm{pH}$ management during hypothermia, the first termed $\mathrm{pH}$ strategy, where blood $\mathrm{pH}$ is maintained constant at 7,40 at all temperatures with $\mathrm{PaCO}_{2}$ adjustment, and in the second type of regulation, termed $\alpha$-stat strategy, the blood pH is increased according to decrease in temperature and the total $\mathrm{CO}_{2}$. content of the blood remain constant. In this present review the relationship between blood $\mathrm{pH}$ and intracelular $\mathrm{pH}$ and the ionic alterations induced by hipothermia are discussed.

DESCRIPTORS: hipothermia, induced, in heart surgery.

\section{REFERÊNCIAS BIBLIOGRÁFICAS}

1 AUSTIN, J. B. \& CULLEN, G. E. - Hydrogen ion concentration of the blood in the health and disease. Medicine, 4: 275-343, 1925.

2 BASHEIN, G.; TOWNES, B. D.; NESSLY, M. L.; BLEDSOE, S. W.; HORNBEIN, T. F.; DAVIS, K. B.; GOLDSTEIN, D. E.; COPPEL, D. B. - A randomized study of carbon dioxide management during hypothermic cardiopulmonary bypass. Anesthesiology, 72: 7-15, 1990.

3 BECKER, H.; VINTEN-JOHANSEN, J.; BUCKBERG, G. D.; ROBERTSON, J. M.; LEAF, J. D.; LAZAR, H. L.; MANGANARO, A. J. - Myocardial damage caused by keeping $\mathrm{pH} 7,40$ during sistemic deep hypothermia. J. Thorac. Cardiovasc. Surg., 82: 810-820, 1981.

4 BELSEY, R. H. R.; DOWLATSHAHI, K.; KEEN, G.; SKINNER, D. B. - Profound hypothermia in cardiac surgery. J. Thorac. Cardiovasc Surg., 56: 497-509, 1968.

5 BOVE, E. L.; WEST, H. L.; PASKANIK, A. M. Hypothermic cardiopulmonary bypass: a comparison between alpha and $\mathrm{pH}$-stat regulation in the dog. J. Surg. Res., 42: 66-73, 1987.

6 BRADLEY, A. F.; STUFFEL, M.; SEVERINGHAUS, J. W. - Effect of temperature on PCO2 and PO2 of blood in vitro. J. Appl. Physiol., 9: 201-204, 1956.

7 DAVIS, B. - On the importance of being ionized. Arch. Biochem. Biophys., 78: 497-509, 1958.

8 ERGIN, M. A.; GALLA, J. D.; LANSMAN, S. L.; QUINTANA, C.; BODIAN, C.; GRIEPP, R. B. Hypothermic circulatory arrest in operations on the thoracic orta. J. Thorac. Cardiovasc. Surg., 107: 788-799, 1994.
9 HENRIKSEN, L. - Brain luxury perfusion during cardiopulmonary bypass in humans: a study of cerebral blood flow response to changes to changes in $\mathrm{CO} 2, \mathrm{O} 2$, and blood pressure. J. Cereb. Blood Flow Metab., 6: 366-378, 1986.

10 HICKEY, P. R. \& ANDERSEN, N. P. - Deep hypothermic circulatory arrest: a review of pathophysiology and clinical experience as a basis for anesthetic management. J. Cardiothorac. Anesth., 1: 137-155, 1987.

11 HOWELL, B. J.; BAUMGARTNER, F. W.; BONDI, K.; RAHN, H. - Acid-base balance in cold-blooded vertebratess as a function of body temperature. Am. J. Physiol., 218: 600-606, 1970.

JONAS, R. A.; BELLINGER, D. C.; RAPPAPORT, L. A.; WERNOVSKY, G.; HICKEY, P. R.; FARREL, D. M.; NEWBURGER, J. W. - Relation of $\mathrm{pH}$ strategy and development outcome after hypothermic circulatory arrest. J. Thorac. Cardiovasc. Surg., 106: 362-368, 1993.

13 KERN, F. H.; UNGERLEIDER, R. M.; QUILL, T. J.; BALDWIN, B.; WHITE, W. D.; REVES, J. G.; GREELEY, W. J. - Cerebral blood flow response to changes in arterial carbon dioxide tension during hypothermic cardiopulmonary bypass in children. J. Thorac. Cardiovasc. Surg., 101: 618-622, 1991.

KOUCHOUKOS, N. T.; WAREING, T. H.; IZUMOTO, H.; KLAUSING, W.; ABBOUD, N. - Elective hypothermic cardiopulmonary bypass and circulatory arrest for spinal cord protection during operations on the thoracoabdominal aorta. J. Thorac. Cardiovasc. Surg., 99: 659-664, 1990.

15 MCCONNELL, D. H.; WHITE, F.; NELSON, R. L.; GOLDSTEIN, S. M.; MALONEY, J. V.; DeLAND, E. 
C.; BUCKBERG, G. D. - Importance of alkalosis in maintenance of "ideal" blood $\mathrm{pH}$ during hypothermia. Surg. Forum, 26: 263-265, 1975.

MATTHEWS, A. S.; STEAD, A. L.; ABBOTT, T. R. Acid-base control during hypothermia. Anaesthesia, 39: 649-654, 1984.

17 MURKIN, J. M.; FARRAR, J. K.; TWEED, W. A.; MCKENZIE, F. N.; GUIRAUDON, G. - Cerebral autoregulation and flow/metabolism coupling during cardiopulmonary bypass: the influence of $\mathrm{PaCO} 2$. Anesth. Analg., 66: 825-832, 1987.

18 MURKIN, J. M.; MARTZKE, J. S.; BUCHAN, A. M.; BENTLEY, C. - pH management during prolonged hypothermic cardiopulmonary bypass significantly influences the incidence of postoperative neuropsychological dysfunction. Can. J. Anaesth., 40: A46, 1993.

NEVIN, M.; COLCHESTER, A. L.; ADAMS, S.; PEPPER, J. R. - Evidence for involvement of hypocapnia and hypoperfusion in aetiology of neurological deficit after cardiopulmonary bypass. Lancet, 2: 1493-1495, 1987.

20 NEVIN, M. \& PEPPER, J. R. - Carbon dioxide, brain damage and cardiac surgery. Lancet, 1: 949, 1988.

21 NIAZI, S. A. \& LEWIS, F. J. - Profound hypothermia in the dog. Surg. Gynecol. Obstet, 102: 98-106, 1956.

NORWOOD, W. I.; NORWOOD, C. R.; CASTANEDA, A. R. - Cerebral anoxia: effect of deep hypothermia and $\mathrm{pH}$. Surgery, 86: 203-209, 1979.

23 PROUGH, D. S.; STUMP, D. A.; ROY, R. C.; GRAVLEE, C. P.; WILLIAMS, T.; MILLS, S. A.; HINSHELWOOD, L.; HOWARD, G. - Response of cerebral blood flow to change in carbon dioxide tension during hypothermic cardiopulmonary bypass. Anesthesiology, 64: 576-581, 1986.

$24 \mathrm{RAHN}, \mathrm{H}$. - Body temperature and acid-base regulation. Pneumologie, 151: 87-94, 1974.

25 RAHN, H.; REEVES, R. B.; HOWELL, B. J. - Hydrogen ion regulation, temperature and evolution. Am. Rev. Respir. Dis., 112: 165-172, 1975.

26 REAM, A. K.; REITZ, B. A.; SILVERBERG, G. - Temperature correction of $\mathrm{PCO} 2$ and $\mathrm{pH}$ in estimating acidbasic status: an example of the emperor's new clothes? Anesthesiology, 56: 41-44, 1982.

REEVES, R. B. - Role of body temperature in determining the acid-base state in vertebrates. Fed. Proc., 28: 1204-1208,., 1969.

REEVES, R. B. - An imidazolic alphastat hypothesis for vertebrate acid-base regulation: tissue carbon dioxide content and body temperature in bullfrogs. Respir. Physiol., 14: 219-236, 1972.
REEVES, R. B. - Temperature-iduced changes in blood acid-base statusp: Donnan $\mathrm{r} \mathrm{Cl}$ and red cell volume. J. Appl. Pysiol, 40: 762-767, 1976.

REEVES, R. B. - Temperature-induced changes in blood acid-base status: $\mathrm{pH}$ and $\mathrm{PaCO} 2$ in a binary buffer. J. Appl. Physiol., 40: 752-761, 1976.

31 ROSENTHAL, T.B. - The effect of temperature on the $\mathrm{pH}$ of blood and plasma in vitro. J. Biol. Chem. 173: $25-30,1948$.

32 SEVERINGHAUS, J. W. - Blood gas calculator. J. Appl. Pysiol., 21: 1108-1116, 1948.

33 SEVERINGHAUS, H. W. - Respiration and hypothermia Ann. N. Y. Acad. Sci., 80: 384-394, 1959.

34 STADIE, W. C.; AUSTIN, J. B.; ROBINSON, H. W. - The effect of temperature on the acid-base proteins equilibrium and its influence on the $\mathrm{CO} 2$ absorptions curve of whole blood, true, and separated plasma. J. Biol. Chem., 66: 901-920, 1925.

35 SVENSSON, L. G.; CRAWFORD, E. S.; HESS, K. R. COSELLI, J. S.; SAFI, H. J. - Deep hypothermia and circulatory arrest: determinants of stroke and early mortality in 656 adult patients. J. Thorac. Cardiovasc. Surg., 106: 19-31, 1993.

36 SWAIN, J. A. - Hypothermia and blood pH. Arch. Intern. Med., 148: 1643-1646, 1988.

37 SWAIN, J. A.; MCDONALD, T. J.; ROBBINS, R. C. HAMPSHIRE, V. A. - Hemodynamics and metabolism during surface induced hypothermia in the dog: a comparison of $\mathrm{pH}$ management strategies. J. Surg. Res., 48: 217-222, 1990.

38 SWAIN, J. A.; WHITE, F. N.; PETERS, R. M. - The effect of $\mathrm{pH}$ on the hypothermic ventricular fibrilation treshold. J. Thorac. Cardiovasc. Surg., 87: 445-451, 1984.

SWAN, H. - The hydroxil-hydrogen ion concentration ratio during hypothermia. Surg. Gynecol. Obstet., 155 $897-912,1982$.

SWAN, H. - The importance of acid-base management for cardiac and cerebral preservation during open heart operations. Surg Gynecol. Obstet., 158: 391 414,1984

41 VENN, G. E.; SHERRY, K.; KLINGER, L.; NEWMAN, S.; HARRISON, M.; ELL, P. J.; TREASURE, T. Cerebral blood flow determinants and their clinical implication during cardiopulmonary bypass. Perfusion, 3: 271-280, 1988.

42 WHITE, F. N. - A comparative physiological approach to hypothermia. J. Thorac. Cardiovasc. Surg., 82: 821828,1981

43 WILLIAMS, J. J. \& MARSHALL, B. E. - A fresh look at an old question. Anesthesiology, 56: 1-2, 1982. 